\title{
NADPH Oxidase Contributes Directly to Oxidative Stress and Apoptosis in Nerve Growth Factor-Deprived Sympathetic Neurons
}

\author{
Steven P. Tammariello, ${ }^{1}$ Mark T. Quinn, ${ }^{2}$ and Steven Estus ${ }^{1}$ \\ ${ }^{1}$ Department of Physiology, Sanders-Brown Center on Aging, University of Kentucky, Lexington, Kentucky 40536, \\ 2Department of Veterinary Molecular Biology, Montana State University, Bozeman, Montana 59717
}

Reactive oxygen species (ROS) are necessary for programmed cell death $(P C D)$ in neurons, but the underlying ROS-producing enzymes have not been identified. NADPH oxidase produces ROS, although the expression of its five subunits are thought to be restricted largely to non-neuronal cells. Here, we show that NADPH oxidase subunits are present in neurons. Moreover, both an NADPH oxidase inhibitor, diphenyleneiodonium, and
NAPDH oxidase genetic deficiency inhibit apoptosis in a classic model of PCD, i.e., NGF-deprived sympathetic neurons. Overall, these results indicate that NADPH oxidase is unexpectedly present in neurons and can contribute to neuronal apoptosis.

Key words: apoptosis; sympathetic neuron; oxidative stress; reactive oxygen species; NADPH oxidase; programmed cell death
Depriving neonatal sympathetic neurons of NGF in vitro leads to neuronal apoptosis in a process that recapitulates the naturally occurring death of these neurons during nervous system maturation (Oppenheim, 1991; Deckwerth and Johnson, 1993; Edwards and Tolkovsky, 1994). Reactive oxygen species (ROS) appear a necessary "trigger" in this apoptotic process, because a burst of oxidative stress is detected $4 \mathrm{hr}$ after NGF withdrawal, and superoxide dismutase (SOD) overexpression inhibits apoptosis (Greenlund et al., 1995; Jordan et al., 1995). This ROS is not sufficient to induce apoptosis, because apoptosis is blocked if sympathetic neurons are exposed after ROS generation to NGF, cAMP, $\mathrm{K}^{+}$depolarization, or inhibitors of RNA or protein synthesis (Deckwerth and Johnson, 1993; Edwards and Tolkovsky, 1994; Franklin et al., 1995). The source of ROS in NGFdeprived sympathetic neurons has not yet been identified.

NADPH oxidase is a five-subunit enzyme that transfers electrons from NADPH to molecular oxygen to produce superoxide radicals and is a key source of microbicidal oxidants in the immune response (DeLeo and Quinn, 1996). Although expression of the NADPH oxidase subunits was thought to be confined to cells of hematopoietic origin, other studies have suggested that NADPH oxidase subunits may also be present in additional cell types (Youngson et al., 1991; Jones et al., 1994). That NADPH oxidase may contribute to neuronal apoptosis has been suggested by findings that NADPH oxidase-deficient mice exhibit reduced injury after stroke (Walder et al., 1997) and that Rac1, known to be required for maximal NADPH oxidase activation (reviewed in Bokoch, 1995), has been implicated in sympathetic neuron apoptosis after NGF deprivation (Bazenet et al., 1998).

Here, we report that NADPH oxidase is present in neurons.

Received May 12, 1999; revised Sept. 28, 1999; accepted Nov. 5, 1999.

This work was supported by National Institutes of Health Grants T32AG-00242 (S.P.T.), R29 NS-35607 (S.E.), and RO1 AR-42426 (M.T.Q.) and an American Heart Association Established Investigator Award (M.T.Q.). We thank H. M. Tucker and E.M. Johnson for helpful discussion.

Correspondence should be addressed to Steven Estus, 800 South Limestone, Lexington, KY 40536-0230. E-mail: sestus@aging.coa.uky.edu.

Dr. Tammariello's present address: Department of Biological Sciences, Binghamton University, Science III, Room 142, Binghamton, NY 13902-6000.

Copyright $\odot 1999$ Society for Neuroscience $0270-6474 / 99 / 200001-05 \$ 15.00 / 0$
Moreover, by using a combination of pharmacological and genetic approaches, we implicate NADPH oxidase as contributing to oxidative stress and apoptosis in a well characterized model of developmentally appropriate neuronal loss, i.e., sympathetic neurons undergoing NGF deprivation-induced apoptosis. Overall, these results provide compelling evidence that NADPH oxidase plays a heretofore unsuspected role early in neuronal programmed cell death.

\section{MATERIALS AND METHODS}

Neuronal cultures and treatments. Primary rodent sympathetic neuron cultures were plated onto laminin-coated tissue culture dishes in NGFcontaining medium as described (Estus et al., 1994). Murine littermates from mice hemizygous or heterozygous for gp91-phox were genotyped for the presence of intact gp91-phox (Pollock et al., 1995). After 3-4 d in culture, NGF deprivation was performed by replacing the NGFcontaining medium with an identical medium, except that NGF was replaced with an NGF-neutralizing antibody (Sigma, St. Louis, MO). Diphenyleneiodonium (DPI; Toronto Research Chemicals, North York, Ontario, Canada) stock solutions (100 mM) were made in DMSO and then added to the culture dishes at the indicated final concentrations. Control cultures were treated with DMSO vehicle only.

$R T$-PCR. Poly $\left(\mathrm{A}^{+}\right)$RNA was prepared from 2000 neurons and converted to cDNA, and specific cDNAs were amplified by subjecting $3 \%$ of the cDNA to 25 PCR cycles as described (Estus et al., 1994). After amplification, cDNAs were separated by PAGE and visualized by using SYBR-gold fluorescent staining (Molecular Probes, Eugene, OR). Primer sequences were gp91-phox sense primer, 5'-GGTTTATGATGATGGGCCTAA-3', and gp91-phox antisense primer, 5'-GCACTGGAACCCCTGAGAAA-3' (158 bp product); $p 22$-phox sense primer, 5'-CCCCGGGGAAAGAGGAAAAA-3', and $p 22$-phox antisense primer, 5'-AGGACAG-

This article is published in The Journal of Neuroscience, Rapid Communications Section, which publishes brief, peerreviewed papers online, not in print. Rapid Communications are posted online approximately one month earlier than they would appear if printed. They are listed in the Table of Contents of the next open issue of JNeurosci. Cite this article as: JNeurosci, 2000, 20:RC53 (1-5). The publication date is the date of posting online at www.jneurosci.org.

http://www.jneurosci.org/cgi/content/full/3845 
CCCGGACGTAGTA-3' (115 bp product); p67-phox sense primer, 5'GGGAACCAGCTGATAGACTA-3', and p67-phox antisense primer, 5'TCCATTCCTCYTTCTTGGCA-3' (105 bp product); p47-phox sense primer, 5'-CAGCCAGCACTATGTGTACA-3', and p47-phox antisense primer, 5'-GAACTCGTAGATCTCGGTGAA-3' (67 bp product); and $p 40$ phox sense primer, 5'-TGGCCCAGCAGCTGCG-3', and p40-phox antisense primer, 5'-CGRTAGCGGCGGTAGATGAG-3' (170 bp product). The identity of the amplified cDNAs was confirmed by direct sequencing.

Immunofluorescence. Cells were fixed with paraformaldehyde, permeabilized with $0.5 \%$ Triton X-100 in PBS, and then incubated with blocking buffer (PBS, 5\% goat serum, and $0.3 \%$ Triton X-100) for $30 \mathrm{~min}$. The cells were then labeled with polyclonal antibodies against NADPH oxidase components (DeLeo and Quinn, 1996) (dilution of 1:500) or a monoclonal antibody against MAP-2 (Sigma) (dilution of 1:400) in blocking buffer for $1 \mathrm{hr}$ at $25^{\circ} \mathrm{C}$. After PBS washes, primary antibodies were detected with a Cy-3-conjugated secondary antibody (Jackson ImmunoResearch, West Grove, PA) and counterstained with Hoechst 33258 (Molecular Probes).

Cell death counts. To assess the frequency of neuronal apoptosis, cultures were treated as described and then fixed with $4 \%$ paraformaldehyde in PBS for $20 \mathrm{~min}$. After staining with Hoechst $33258(1 \mu \mathrm{g} / \mathrm{ml}$ in PBS) for $10 \mathrm{~min}$, the frequency of neurons manifesting condensed or punctate chromatin was scored by an observer "blinded" as to the neuron treatments. Each condition was evaluated in at least duplicate wells, with at least 200 neurons scored in each well. Differences were analyzed statistically by using a two-tailed Student's $t$ test (Statview; Abacus Concepts, Calabasas, CA).

Oxidative stress measurements. Oxidative stress was assessed by using the indicator $2^{\prime}, 7^{\prime}$-dichlorodihydrofluoroscein diacetate $\left(\mathrm{H}_{2} \mathrm{DCFDA}\right.$; Molecular Probes); this compound is converted to the fluorescent $2^{\prime}, 7^{\prime}$ dichlorofluoroscein (DCF) after oxidation as well as intracellular esterase cleavage of the acetate groups. Because changes in cellular $\mathrm{pH}$ or other events may modulate DCF fluorescence in an artifactual manner, we also examined the effects of DPI on the fluorescence observed in cells treated with 5-carboxy-2',7'-dichlorofluoroscein diacetate (DCFDA; Molecular Probes); cytosolic esterases hydrolyze the acetate groups of DCFDA, converting it directly to the fluorescent DCF without requiring oxidation. Neurons were plated onto polyornithine-laminin-coated 96 well tissue culture dishes at a density of $\sim 500$ neurons per well. After 5-6 $\mathrm{d}$ in culture, the neurons were either maintained or deprived of NGF in the presence or absence of DPI $(0.5 \mu \mathrm{M})$ for $3.5 \mathrm{hr}$. DCF was then added to a final concentration of $1 \mathrm{~mm}$, and the dishes were returned to the $37^{\circ} \mathrm{C}$ incubator for $30 \mathrm{~min}$. Fluorescence was then measured by excitation at $485 \mathrm{~nm}$ and emission at $535 \mathrm{~nm}$ by using a Perkin-Elmer (Norwalk, CT) HTS-7000 plate reader. Because of potential oxidation by the excitation laser, samples were scanned only once. Assays were performed in pentuplicate.

\section{RESULTS}

\section{NADPH oxidase is present at the mRNA and protein levels}

To determine whether NADPH oxidase is expressed in sympathetic neurons, we examined the expression of NADPH oxidase subunits at the mRNA and protein levels. The mRNAs encoding each of the five subunits were detected in RNA isolated from sympathetic neuron preparations (Fig. $1 A$ ). Because these neuronal preparations are $\sim 5 \%$ non-neuronal cells (Schwann cells and fibroblasts), we repeated this analysis on RNA purified from PC12 cells, which are a homogeneous cell line with characteristics of sympathoadrenal precursors. All five mRNAs encoding the NADPH oxidase subunits were also present in PC12 cells (data not shown). To examine protein expression, we performed immunofluorescence by using available antibodies produced against three of the NADPH oxidase subunits (DeLeo and Quinn, 1996). Each of the NADPH oxidase subunits examined, i.e., p67-phox, p47-phox, and p40-phox, were present in sympathetic neurons at the protein level (Fig. $1 B$ ). This work confirms and dramatically extends that of others that showed that one NADPH oxidase subunit mRNA is expressed in neurons (Mizuki et al., 1998).
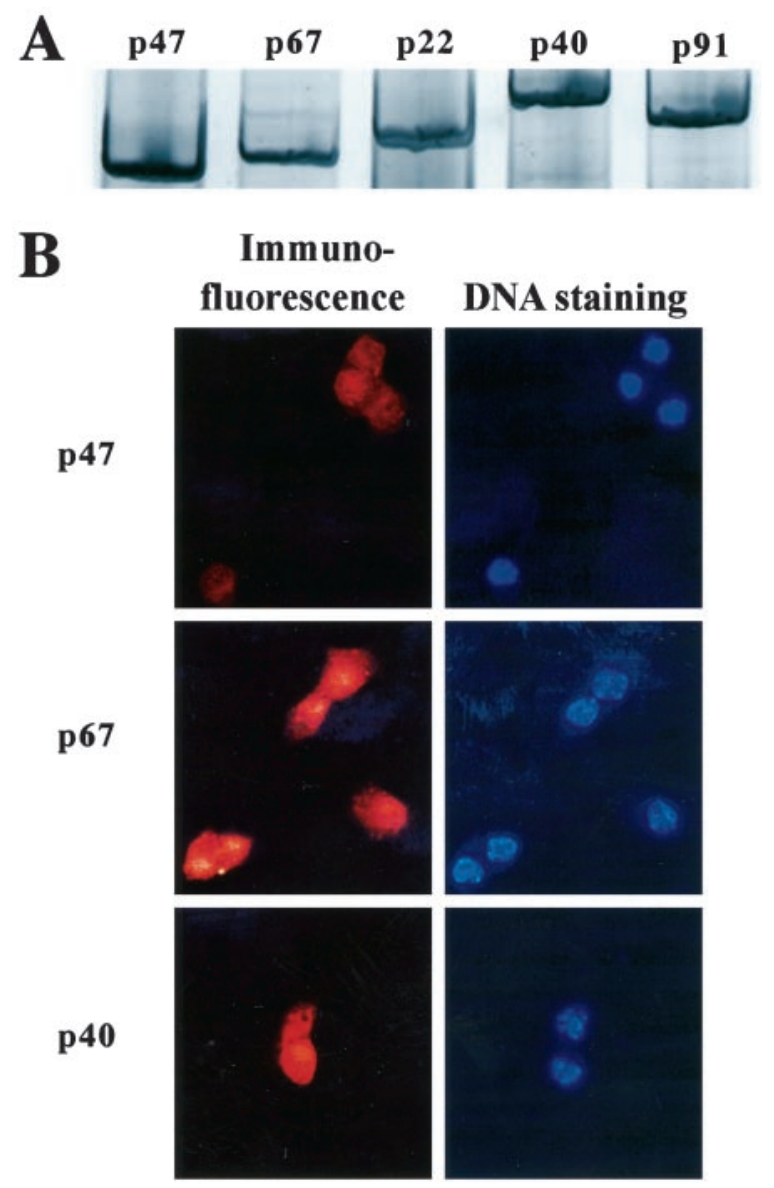

Figure 1. NADPH oxidase subunits are present in neurons at the mRNA $(A)$ and protein $(B)$ levels. The expression of each NADPH oxidase subunit at the mRNA level was detected by performing 25 PCR cycles on $3 \%$ of the cDNA generated from RNA isolated from $\sim 2000$ neurons $(A)$. To detect the expression of the indicated NADPH oxidase subunits at the protein level, neurons were labeled with antibodies against the three cytosolic subunits; bound antibodies were then detected with a $\mathrm{Cy}-3-$ labeled secondary antibody $(B)$. Similar results were obtained with monoclonal antibodies raised against these same phox subunits (data not shown). Hoechst 33258 staining was performed in parallel as a counterstain to reveal nuclei. No staining was observed in neurons stained with the secondary antibody alone.

\section{DPI inhibits NGF deprivation-induced sympathetic neuron death in a concentration- and time-dependent manner}

To begin to evaluate the NADPH oxidase role in neuronal apoptosis, we analyzed whether an NADPH oxidase inhibitor, DPI, protected sympathetic neurons from death after NGF deprivation. Neurons were treated with DPI coincident with NGF withdrawal and apoptosis detected subsequently by using Hoechst 33258 staining to visualize chromatin condensation. Sympathetic neurons began to exhibit chromatin condensation $24 \mathrm{hr}$ after NGF withdrawal (Fig. 2A,C,G) (Deckwerth and Johnson, 1993; Edwards and Tolkovsky, 1994), which appeared largely blocked by DPI treatment (Fig. $2 E$ ). Indeed, when this effect was quantified, DPI was found to provide concentration-dependent neuroprotection, reducing the frequency of apoptosis to that of neurons maintained in the presence of NGF at concentrations of $0.5-1 \mu \mathrm{M}$ (Fig. 2G). The neuroprotective DPI concentrations are essentially identical to those used by others to inhibit NADPH oxidase (Lo 

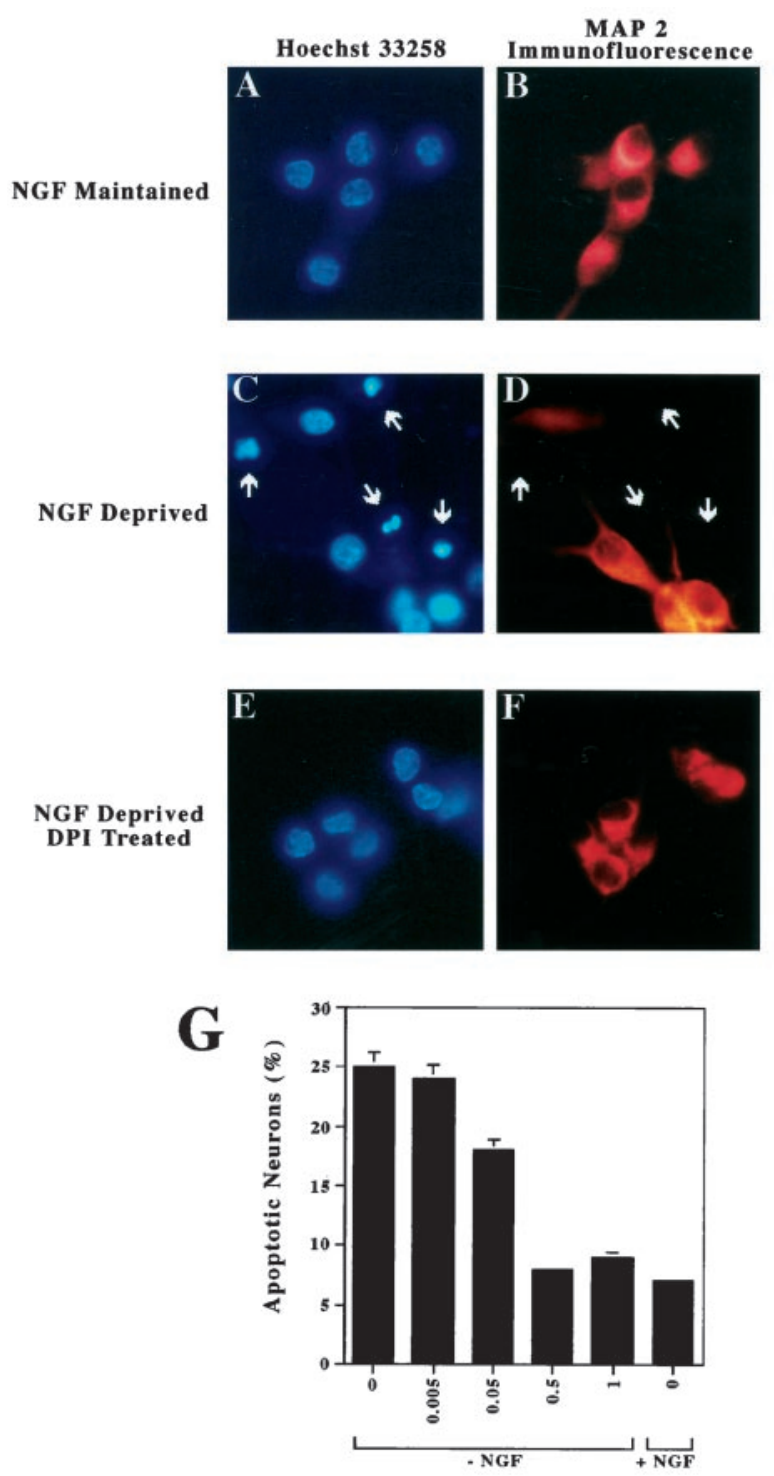

[DPI] $(\boldsymbol{\mu} \mathbf{M})$

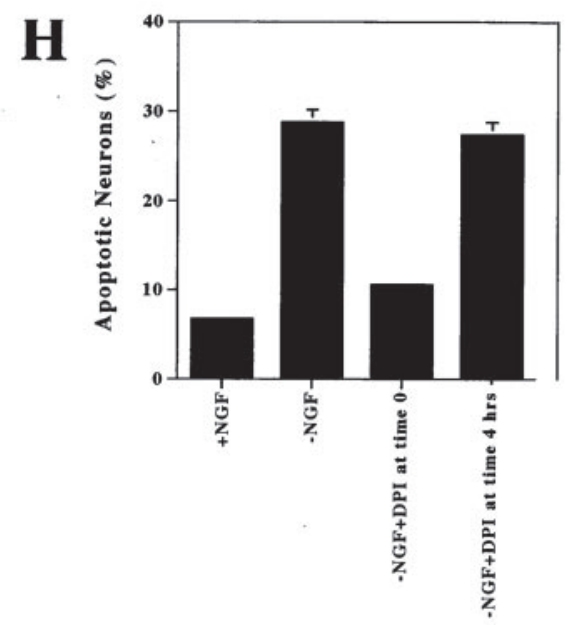

Figure 2. DPI inhibits NGF deprivation-induced sympathetic neuron death. Sympathetic neurons were deprived of NGF in the presence or absence of DPI $(0.5 \mu \mathrm{M})$ for $24 \mathrm{hr}(A-F)$. The neurons were then fixed and stained with Hoechst 33258 to discern chromatin integrity $(A, C, E)$ or with an antibody against MAP-2 to assess cytoskeletal integrity $(B, D, F)$. et al., 1998). Neuronal cytoskeletal integrity was also evaluated indirectly by using immunofluorescence to visualize MAP-2. Robust MAP-2 staining is observed in NGF-maintained neurons (Fig. $2 B$ ) but was barely detected in neurons undergoing chromatin condensation (Fig. 2D). DPI treatment blocked this apoptosis-associated loss in MAP-2 staining (Fig. $2 F$ ).

Because oxidative stress appears confined to the first $4 \mathrm{hr}$ of NGF deprivation (Greenlund et al., 1995), we also evaluated whether the critical period of DPI neuroprotective action coincides with this interval. Neurons were treated with DPI either coincident with NGF deprivation or $4 \mathrm{hr}$ later, and the frequency of apoptosis was scored at $24 \mathrm{hr}$ of deprivation. DPI was neuroprotective only if neurons were treated coincident with NGF deprivation (Fig. $2 H$ ). Hence, the timing of DPI actions is consistent with DPI acting to inhibit ROS generation. In addition, these results indicate that DPI is likely not inhibiting apoptosis by directly inhibiting RNA or protein synthesis, or caspases, or by increasing $\mathrm{Ca}^{2+}$ or cAMP, because agents with these actions block death even if treatments are initiated much later than $4 \mathrm{hr}$ after NGF deprivation (Deckwerth and Johnson, 1993; Edwards and Tolkovsky, 1994; Franklin et al., 1995; Deshmukh et al., 1997). In summary, DPI treatment protects sympathetic neurons from NGF deprivation-induced apoptosis in a time- and concentration-dependent manner.

\section{DPI blocks NGF deprivation-induced oxidative stress}

To evaluate directly whether DPI inhibited oxidative stress induced by NGF deprivation, neurons were deprived of NGF in the presence or absence of DPI for $4 \mathrm{hr}$. During the last $30 \mathrm{~min}$ of this interval, the neurons were loaded with $\mathrm{H}_{2} \mathrm{DCFDA}$, which is converted to the fluorescent DCF by oxidative stress. Changes in DCF fluorescence were then quantified by using a fluorescent microtiter plate reader, which greatly facilitated the assay, as opposed to using confocal microscopy and quantifying individual neurons, as reported by others previously (Greenlund et al., 1995; Dugan et al., 1997). NGF deprivation induced an almost twofold increase in DCF fluorescence, which DPI significantly inhibited (Fig. $3 A ; p<0.05$, Student's $t$ test). DPI had no effect on DCF values in NGF-maintained neurons (Fig. $3 A$ ). Because changes in cellular $\mathrm{pH}$ can modulate DCF fluorescence in an artifactual manner, we also quantified DPI effects on the fluorescence observed in cells treated with DCFDA; this compound is converted directly to fluorescent DCF by cytosolic esterases, independent of oxidation. Neither DPI nor NGF deprivation altered the fluorescence of neurons loaded with this dye (Fig. $3 B$ ). Hence, DPI blocks the oxidative stress induced by NGF deprivation. Because oxidative stress is necessary for apoptosis in this paradigm (Greenlund et al., 1995; Jordan et al., 1995), DPI inhibition of oxidative stress may account for DPI inhibition of apoptosis.

\section{$\leftarrow$}

Chromatin condensation and disappearance of MAP-2 staining are concomitant $(C, D$, arrows). These concentration-dependent neuroprotective effects were quantified by depriving neurons of NGF in the presence of the indicated concentrations of DPI for $24 \mathrm{hr}$. The chromatin integrity of the neurons was then scored by an observer blinded as to the nature of the cellular treatments $(G)$. These data represent the mean \pm SD of three experiments each performed in duplicate. To identify the critical period of DPI actions, neurons were treated with DPI $(0.5 \mu \mathrm{M})$ either coincident with NGF deprivation or $4 \mathrm{hr}$ after NGF deprivation. Chromatin integrity was then scored at $24 \mathrm{hr}$ after NGF deprivation $(H)$. These data represent the mean \pm range of two experiments each performed in triplicate. 
A.

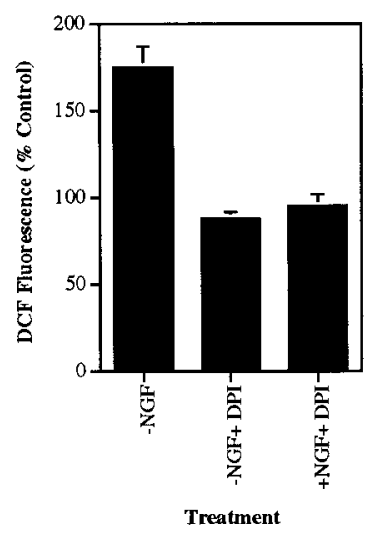

B.

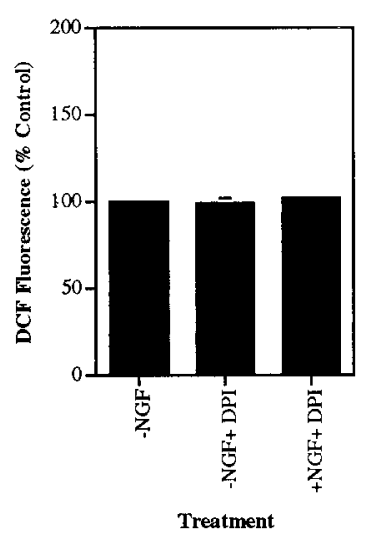

Figure 3. DPI blocks NGF deprivation-induced oxidative stress. Neurons were treated with DPI or NGF deprivation as indicated for $3.5 \mathrm{hr}$. They were then loaded for $30 \mathrm{~min}$ with either $\mathrm{H}_{2}$ DCFDA, to assess oxidative stress $(A)$, or DCFDA, to assess whether DCF fluorescence was altered artifactually by DPI $(B)$. DCF fluorescence was then quantified by using a fluorescent microtiter plate reader. The data in $A$ represent the mean $\pm \mathrm{SE}$ of four separate experiments, whereas the data in $B$ represent the mean \pm range of two separate experiments.

\section{Apoptosis is decreased in neurons lacking NADPH oxidase activity}

Although DPI is known to inhibit NADPH oxidase, DPI is also capable of inhibiting additional flavin-dependent enzymes, including nitric oxide synthase, NADPH-cytochrome $\mathrm{P} 450$ reductase, and NADH-ubiquinone oxireductase (Stuehr et al., 1991; Li and Trush, 1998; McGuire et al., 1998). Therefore, to more rigorously evaluate the role of NADPH oxidase in neuronal death, we chose a nonpharmacological means to inhibit NADPH oxidase in neuronal death, i.e., genetic deletion. We obtained mice deficient in gp91-phox, one of the catalytic NADPH oxidase subunits necessary for superoxide production (Pollock et al., 1995). Sympathetic neuronal cultures were established from neonatal littermates that were wild-type or deficient with respect to gp91-phox and subjected to NGF deprivation in parallel. Although neurons from wild-type mice died at rates comparable with those of rat neurons, the frequency of apoptosis in neurons isolated from gp91-phox-deficient mice was equivalent to that of NGF-maintained neurons (Fig. 4). Hence, two separate means of inhibiting NADPH oxidase both implicate this enzyme as contributing to NGF deprivation-induced neuronal apoptosis.

\section{DISCUSSION}

The significance of the results reported here are twofold. First, NADPH oxidase subunits appear present in neurons at the mRNA and protein levels. Because NADPH oxidase contributes to oxidative stress in other tissues, these results identify NADPH oxidase as a previously unconsidered source of neuronal oxidative stress. Second, this report provides compelling evidence that NADPH oxidase contributes to the ROS and apoptosis that are induced in a classic programmed cell death model, i.e., NGFdeprived sympathetic neurons. Because oxidative stress appears to play an important role in many cell death paradigms associated with neurodegenerative diseases, such as Alzheimer's disease, amyotrophic lateral sclerosis, and stroke (for review, see Cotman, 1998; Facchinetti et al., 1998; Sun and Chen, 1998; Lee et al., 1999), that NADPH oxidase is present in neural tissue and

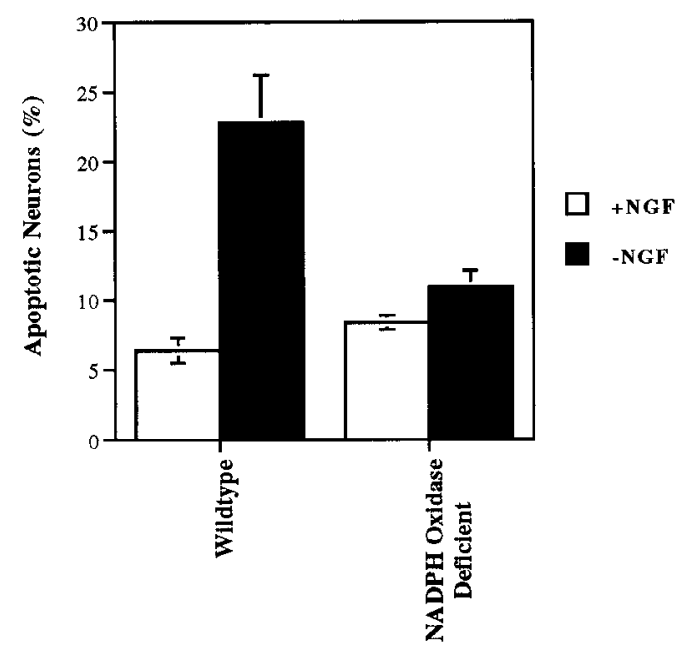

Figure 4. NADPH oxidase-deficient neurons are resistant to NGF deprivation-induced apoptosis. Neurons from wild-type or NADPH oxidase-deficient neonatal littermates were prepared and maintained in vitro for $3 \mathrm{~d}$. They were then deprived of NGF for $24 \mathrm{hr}$, and the frequency of apoptotic neurons was determined by blinded scoring of Hoechst 33258 staining. These results represent the mean $\pm \mathrm{SE}$ of four separate experiments.

contributes to neuronal death may have implications beyond the sympathetic neuron model.

The mechanisms leading to NADPH oxidase activation in neurons are unclear, but results from other cell types provide some insights. In resting lymphocytes, NADPH oxidase subunits are segregated into a three-member cytosolic component (p40phox, p47-phox, and p67-phox) and a two-member plasma membrane component (p22-phox and gp91-phox) (DeLeo and Quinn, 1996). NADPH oxidase is activated when the cytosolic subunits translocate to the membrane where multiple binding interactions occur among the five subunits (DeLeo and Quinn, 1996). Translocation and assembly requires p47-phox phosphorylation, although the relevant kinases have not yet been identified (Johnson et al., 1998). Maximal NADPH oxidase activation is dependent on a low molecular weight GTP-binding protein, Rac1, joining the complex (Bokoch, 1995). Interestingly, Bazenet et al. (1998) recently reported that overexpression of constitutively active Rac1 as well as the related CDC42 induces apoptosis in NGFmaintained sympathetic neurons and that overexpression of a Rac1 or CDC42 dominant negative gene blocks apoptosis in NGF-deprived neurons. Hence, NADPH oxidase activation after NGF deprivation may be regulated in part by Rac1 and/or CDC42 as well as unknown kinase(s).

Several lines of evidence suggest that NADPH oxidase may be a useful therapeutic target, especially in relatively acute situations of neuronal loss. For example, Walder et al. (1997) showed that mice lacking NADPH oxidase are resistant to neuronal loss after ischemia. Here, we have used a well established paradigm of trophic factor deprivation to show that NADPH oxidase appears necessary for ROS generation and apoptosis. Whether NADPH oxidase inhibition may be applicable in chronic situations is unclear, because DPI treatment or NADPH oxidase deficiency blocked apoptosis for only 72-96 hr, after which the neurons did begin to die (data now shown); although these results are consistent with those of SOD-protected neurons, which also underwent a delayed apoptosis after NGF deprivation (Greenlund et al., 1995), we interpret these results as suggesting that NADPH 
oxidase inhibition may not be useful in chronic neurodegenerative conditions. That NADPH oxidase may be inhibited at least in the short term without an overly deleterious result is suggested by the observations that mice or humans deficient in NADPH oxidase develop normally but with an increased susceptibility to infection (Pollock et al., 1995; Segal, 1996). Hence, inhibition of NADPH oxidase may provide an effective means to inhibit neuronal loss in acute situations.

In summary, we report that NADPH oxidase subunits are present in neurons. Moreover, both an NADPH oxidase pharmacological inhibitor and NAPDH oxidase genetic deficiency inhibit the death of NGF-deprived sympathetic neurons. Overall, we interpret these results as indicating that NADPH oxidase is unexpectedly present in neurons and can contribute to neuronal apoptosis.

\section{REFERENCES}

Bazenet CE, Mota MA, Rubin LL (1998) The small GTP-binding protein $\mathrm{Cdc} 42$ is required for nerve growth factor withdrawal-induced neuronal death. Proc Natl Acad Sci USA 95:3984-3989.

Bokoch GM (1995) Regulation of the phagocyte respiratory burst by small GTP-binding proteins. Trends Cell Biol 5:109-113.

Cotman CW (1998) Apoptosis decision cascades and neuronal degeneration in Alzheimer's disease. Neurobiol Aging 19:S29-S32.

Deckwerth TL, Johnson Jr EM (1993) Temporal analysis of events associated with programmed cell death (apoptosis) of sympathetic neurons deprived of nerve growth factor (NGF). J Cell Biol 123:1207-1222.

DeLeo FR, Quinn MT (1996) Assembly of the phagocyte NADPH oxidase: molecular interaction of oxidase proteins. J Leukoc Biol 60:677-691.

Deshmukh D, Vasilakos J, Deckwerth TL, Lampe PA, Shivers BD, Johnson EM (1997) Genetic and metabolic status of NGF-deprived sympathetic neurons saved by an inhibitor of ICE-family protease. J Cell Biol 135:1341-1354.

Dugan LL, Creedon DJ, Johnson EM, Holtzman DM (1997) Rapid suppression of free radical formation by nerve growth factor involves the mitogen-activated protein kinase pathway. Proc Natl Acad Sci USA 94:4086-4091.

Edwards SN, Tolkovsky AM (1994) Characterization of apoptosis in cultured rat sympathetic neurons after nerve growth factor withdrawal. J Cell Biol 124:537-546.

Estus S, Zaks W, Freeman R, Gruda M, Bravo R, Johnson E (1994) Altered gene expression in neurons during programmed cell death: identification of c-jun as necessary for neuronal apoptosis. J Cell Biol 127:1717-1727.

Facchinetti F, Dawson VL, Dawson TM (1998) Free radicals as mediators of neuronal injury. Cell Mol Neurobiol 18:667-682.

Franklin JL, Sanz-Rodriguez C, Juhasz A, Deckwerth TL, Johnson EM (1995) Chronic depolarization prevents programmed death of sympathetic neurons in vitro but does not support growth: requirement for $\mathrm{Ca}^{2+}$ influx but not Trk activation. J Neurosci 15:643-664.
Greenlund LJS, Deckwerth TL, Johnson EM (1995) Superoxide dismutase delays neuronal apoptosis: a role for reactive oxygen species in programmed neuronal death of protects sympathetic neurons from NGF deprivation induced apoptosis. Neuron 14:303-315.

Johnson JL, Park JW, Benna JE, Faust LP, Inanami O, Babior BM (1998) Activation of p47(PHOX), a cytosolic subunit of the leukocyte NADPH oxidase. Phosphorylation of Ser-359 or Ser-370 precedes phosphorylation at other sites and is required for activity. J Biol Chem 273:35147-35152.

Jones SA, Wood JD, Coffey MJ, Jones OTG (1994) The functional expression of p47-phox and p67-phox may contribute to the generation of superoxide by an NADPH oxidase-like system in human fibroblasts. FEBS Lett 355:178-182.

Jordan J, Ghadge GD, Prehn JH, Toth PT, Roos RP, Miller RJ (1995) Expression of human copper/zinc-superoxide dismutase inhibits the death of rat sympathetic neurons caused by withdrawal of nerve growth factor. Mol Pharmacol 47:1095-1100.

Lee JM, Zipfel GJ, Choi DW (1999) The changing landscape of ischaemic brain injury mechanisms. Nature 399:A7-A14.

Li Y, Trush MA (1998) Diphenyleneiodonium, an NAD(P)H oxidase inhibitor, also potently inhibits mitochondrial reactive oxygen species production. Biochem Biophys Res Commun 253:295-299.

Lo YC, Conquer JA, Grinstein S, Cruz TF (1998) Interleukin-1 $\beta$ induction of c-fos and collagenase expression in articular chondrocytes: involvement of reactive oxygen species. J Cell Biochem 69:19-29.

McGuire JJ, Anderson DJ, McDonald BJ, Narayanasami R, Bennett BM (1998) Inhibition of NADPH-cytochrome P450 reductase and glyceryl trinitrate biotransformation by diphenyleneiodonium sulfate. Biochem Pharmacol 56:881-893.

Mizuki K, Kadomatsu K, Hata K, Ito T, Fan QW, Kage Y, Fukumaki Y, Sakaki Y, Takeshige K, Sumimoto H (1998) Functional modules and expression of mouse p40(phox) and p67(phox), SH3-domain-containing proteins involved in the phagocyte NADPH oxidase complex. Eur J Biochem 251:573-582.

Oppenheim RW (1991) Cell death during development of the nervous system. Annu Rev Neurosci 14:453-501.

Pollock JD, Williams DA, Gifford MA, Li LL, Du X, Fisherman J, Orkin SH, Doerschuk CM, Dinauer MC (1995) Mouse model of X-linked chronic granulomatous disease, an inherited defect in phagocyte superoxide production. Nat Genet 9:202-209.

Segal AW (1996) The NADPH oxidase and chronic granulomatous disease. Mol Med Today 2:129-135.

Stuehr DJ, Fasehun OA, Kwon NS, Gross SS, Gonzalez JA, Levi R, Nathan CF (1991) Inhibition of macrophage and endothelial cell nitric oxide synthase by diphenyleneiodonium and its analogs. FASEB J 5:98-103.

Sun AY, Chen YM (1998) Oxidative stress and neurodegenerative disorders. J Biomed Sci 5:401-414.

Walder CE, Green SP, Darbonne WC, Mathias J, Rae J, Dinauer MC, Curnutte JT, Thomas GR (1997) Ischemic stroke injury is reduced in mice lacking a functional NADPH oxidase. Stroke 28:2252-2258.

Youngson C, Nurse H, Yeger H, Cutz E (1991) Oxygen sensing in airway chemoreceptors. Nature 365:153-155. 